collapse by gravity. Although just a constant of integration in his equations, it represents a repulsive force whose introduction Einstein later regarded as the biggest blunder of his career. But as Peebles pointed out, a non-zero $\Lambda$ produces a healthy increase in the volume available to a given distance, even in a flat universe, and, like it or not, can resolve much of the galaxy-count/redshift dilemma.

Many cosmologists at the meeting shook their heads: adjusting the cosmological geometry to get out of the mess is regarded as the astronomer's last resort. So, is the choice only between a weirder cosmological model than even Einstein wanted and a rather contrived Universe in which galaxies are miraculously consumed by some cosmic vacuum cleaner? It may well be that the resolution of the faintgalaxies dilemma requires a mixture of more reasonable adjustments: a pinch of large-scale structure, a dab of merging and bursting and maybe even some traditional luminosity evolution. The participants agreed that this would be the less interesting outcome. More exciting is the possibility that the most elementary cosmological test fails because of some important and hitherto undiscovered, property of galaxies and the Universe.

Richard S. Ellis and Carlos S. Frenck are in the Department of Physics, University of Durham, Durham DH1 3LE, UK.

\title{
If it smells like a unicorn . . .
}

\section{F. W. Stahl}

In the latest issue of Genetics, Barry Hall ${ }^{1}$ describes new evidence for environmentally directed point mutations in bacteria. Hall's work extends and strengthens that of Cairns, Overbaugh and Miller ${ }^{2}$, who concluded that a strain of the bacterium Escherichia coli unable to use lactose $\left(\mathrm{Lac}^{-}\right)$had a high rate of reversion to $\mathrm{Lac}^{+}$ when placed on a lactose medium. Hall's new evidence on point mutations ${ }^{\prime}$ is important because many readers thought that Cairns et al. had not adequately supported their conclusions (see, for example, the Scientific Correspondence in Nature 336, 525-528; 1988). Hall's earlier work ${ }^{3}$, which showed that a mutation allowing salicin use by $E$. coli occurred only when salicin was in the medium, was also challenged. His mutation to salicin utilization involved the excision of a transposon, and could be regarded as a special case ${ }^{4}$.

The idea that mutations occur without regard to their immediate usefulness has long been central to our view of evolution. So central, in fact, that both Cairns and Hall must have been reminded what happens to him who strikes the king a nonlethal blow. Although none of the critics convincingly dispatched Cairns's contention, some did give comfort to Defenders of the Faith by pointing out that one aspect or another of the reported work could be variously interpreted ${ }^{5}$. The number and variety of these attacks amplified the admission by Cairns et al. ${ }^{2}$ that their paper may have been somewhat unpersuasive.

Some commentators missed the point entirely, arguing that the paper by Cairns et al., by a supposed implication of purpose behind directed mutation, challenged the idea of evolution by natural selection (ref. 6 , countered in ref. 7). Further, the point of the paper was not that nondividing bacteria can mutate. That conclusion had been reached earlier ${ }^{8}$, eliminating trivial explanations ${ }^{9.10}$. The point was that the rate of mutations of a beneficial type was higher than the rate of mutation of a type that was adaptively neutral under the conditions of the test.

The neutral phenotype chosen by Cairns et al. was resistance to growth inhibition by valine. Because this measurement can be made on almost any $E$. coli, the authors were not inconvenienced by having to construct special strains for their work. But comparing one adaptively relevant mutation (reversion to $\mathrm{Lac}^{+}$) to one irrelevant one (mutation to $\mathrm{Val}^{\mathrm{R}}$ ) did not seem adequate to some critics. Cairns et al. assumed that $\mathrm{Lac}^{+}$cells arose in response to energy starvation in the presence of lactose in a special, adaptive way, but the failure of $\mathrm{Val}^{\mathrm{R}}$ mutants to arise was more or less characteristic of mutants in general. With comparable economy, however, the observation could be taken to mean that $\mathrm{Val}^{\mathrm{R}}$ mutants were special, whereas $\mathrm{Lac}^{+}$mutants were behaving in the manner typical of most other mutants occurring, but not measured, in the same experiments.

Hall's new contribution' not only eliminates that particular possibility but offers interesting new kinds of data. First, Hall generalizes the result of Cairns et al. by showing that an amino-acid auxotroph $\left(\operatorname{Trp}^{-}\right)$will revert to prototrophy on a growth medium lacking tryptophan. On the same medium, $\mathrm{Val}^{\mathrm{R}}$ mutants do not arise. Hall then shows that a Cys mutant reverts on a medium lacking cysteine. The double auxotrophic strain, Trp $\mathrm{Cys}^{-}$, provided the material for the critical test of specificity. The double mutant reverts only to $\operatorname{Trp}^{+}$when the culture is starved of tryptophan and only to $\mathrm{Cys}^{+}$when starved of cysteine. Thus, each mutant character reverts when the bacteria can profit from the reversion, but neither character reverts when the reversion would confer no immediate growth advantage.

Cairns, too, has done new experiments that strengthen the argument for directed mutation. An unfortunate feature of the paper of Cairns et al. ${ }^{2}$ was the wimpy mutational response of the $l a c$ amber strain used in the studies. Now Foster and Cairns have repeated those studies using frameshift mutations in lac (personal communication). Some of these mutant alleles revert like gangbusters on medium containing lactose as the only energy source, while mutating at a rate too low to measure accurately when the cells are grown on other energy sources. This robust adaptive mutational response has facilitated the application of more direct methods of measurement, allowing Foster and Cairns to exclude even the most perverse of alternative interpretations. Neither Hall nor Foster and Cairns have demonstrated a molecular mechanism for environmentally directed mutagenesis. But both sets of authors have promised to test extant models, one of which is proffered by Hall', in the near future.

In the distant past, Ryan ${ }^{x-11}$ reported reversion of a His mutant starving for histidine. (Those mutants were conveniently detected as colonies arising days after a His ${ }^{-}$culture was plated on medium lacking histidine.) Had Ryan looked at some adaptively irrelevant mutant type, instead of $\mathrm{His}^{+}$, in the histidine-starved culture, he would have seen that nondividing cells do not mutate at a rate detectable by his methods. Had he looked both at $\mathrm{His}^{+}$and at this other mutant type. he might have recalled Max Delbrück's warning of 1946, which was delivered to André Lwoff at a symposium attended by Ryan": "In the case of mutations of bacteria ... to phage resistance ... the phage does not cause the mutations. In your case of mutations permitting the mutants to utilize succinate ... as sole carbon and energy source ... it [is] an obvious question to ask whether this particular medium had an influence on the mutation rate ... One should keep in mind the possible occurrence of specifically induced adaptive mutations."

F.W. Stahl is in the Institute of Molecular Biology, University of Oregon, Eugene, Oregon 97403-1229, USA.

$$
\begin{array}{l|l}
\text { Cys }^{-}, & \\
\text {test } & \\
\text { verts } & \\
\text { ed of } & 1 \\
\text { rved } & \\
\text { acter } &
\end{array}
$$

1. Hall, B.G. Genetics 126, 5-16 (1990)

Cairns, J. et al. Nature 335. 142-145 (1988)

Hall, B.G. Genetics 120, 887-897(1988)

Symonds, N. Nature 337, 119-120 (1989).

5. Lenski, R.E., Slatkin, M. \& Ayala. F.J. Proc. natn. Acad Lenski. R.E., Slatkin, M. \& Ayala, F.J.
Sci. U.S.A. 86, 2775-2778 (1989).

6. Rees-Mogg. W. Independent. 13 Sept. 1988.

7. Maynard Smith, J. Nature 336, 107-108 (1988).

8. Ryan, F. Proc int. Genet. Symp. 1956, 555-558(1957)

Ryan, F.J. J. gen. Microbiol. 21. 530-549 (1959).

10. Ryan, F.J., Nakada, D. \& Schneider, M.J. Z. Vererblehre 92. 38-4: (1961).

11. Delbrück. M. Cold Spring Harb. Symb. quant. Biol. 11. 139-155 (1947). 